\title{
Synthesis and in vitro antineoplastic evaluation of silver nanoparticles mediated by Agrimoniae herba extract
}

This article was published in the following Dove Press journal:

International Journal of Nanomedicine

15 April 2014

Number of times this article has been viewed

\author{
Ding $\mathrm{Qu}^{1}{ }^{1, *}$ \\ Wenjie Sun ${ }^{1,2, *}$ \\ Yan Chen ${ }^{1,2}$ \\ Jing Zhou' \\ Congyan Liu'
}

'Key Laboratory of New Drug

Delivery System of Chinese Materia Medica, Jiangsu Provincial Academy

of Chinese Medicine, ${ }^{2}$ Department of Pharmaceutics, School of Pharmacy,

Nanjing University of Chinese

Medicine, Nanjing, People's Republic of China

*These authors contributed equally to this work
Correspondence: Yan Chen

Key Laboratory of New Drug Delivery System of Chinese Materia Medica, Jiangsu Provincial Academy of Chinese Medicine, I00 Shizi Road, Nanjing, Jiangsu 210028, People's Republic of China

Tel +862585608672

Fax +862585637809

Email ychen202@hotmail.com

\begin{abstract}
A rapid synthesis of silver nanoparticles (AgNPs) using Agrimoniae herba extract as reducing agent and stabilizer ( $A$. herba-conjugated AgNPs [AH-AgNPs]) were designed, characterized, and evaluated for antitumor therapy feasibility. In this study, critical factors in the preparation of silver nanoparticles, including extraction time, reaction temperature, the concentration of $\mathrm{AgNO}_{3}$, and $A$. herba extract amount, were investigated using ultravioletvisible spectroscopy. AH-AgNPs with well-defined spherical shape, homogeneous distributional small size $(30.34 \mathrm{~nm})$, narrow polydispersity index (0.142), and high negative zeta potential $(-36.8 \mathrm{mV})$ were observed by transmission electron microscopy and dynamic light scattering. Furthermore, the results of X-ray diffraction and Fourier-transform infrared spectroscopy further indicated successful preparation of AH-AgNPs. Acceptable long-term storage stability of AH-AgNPs was also confirmed. More importantly, AH-AgNPs displayed significantly higher antiproliferative effect against a human lung carcinoma cell line (A549 cells) compared with $A$. herba extract and bare AgNPs prepared by sodium citrate. The half-maximal inhibitory concentrations of AH-AgNPs, bare AgNPs, and A. herba extract were $38.13 \mu \mathrm{g} \cdot \mathrm{mL}^{-1}$, $184.87 \mu \mathrm{g} \cdot \mathrm{mL}^{-1}$, and $1.147 \times 10^{4} \mu \mathrm{g} \cdot \mathrm{mL}^{-1}$, respectively. It is suggested that AH-AgNPs exhibit a strong antineoplastic effect on A549 cells, pointing to feasibility of antitumor treatment in the future.
\end{abstract}

Keywords: rapid synthesis, Agrimoniae herba extract, silver nanoparticles, A549 cells, antitumor

\section{Introduction}

As a consequence of their unique physical and surface properties, silver nanoparticles (AgNPs) have been shown to have a wide range of applications in the fields of catalysis, photonics, biology, pharmaceutics, and drug-delivery systems in recent years. ${ }^{1-4}$ In the clinic, AgNPs offer an alternative strategy to reverse the increasing spread of multidrug resistance resulting from the abuse of antibiotics. ${ }^{5-9}$ Notably, AgNPs have also received great attention because of their antimicrobial and anticancer activities. ${ }^{10-12}$ Owing to these distinct functions, a myriad of studies have emerged that focus on the rapid and efficient synthesis of AgNPs for incorporation into medical dressings and devices in the past few decades. ${ }^{13-16}$ Compared with some chemical and physical methods, natural systems capable of reducing silver ions and fabricating NPs at moderate conditions exhibit the promise of generating large amounts of AgNPs with limited energy input and impact on the environment. ${ }^{17}$ To date, bacteria, fungi, and plant extracts have been applied to establish silver nanosized systems, and such synthetic AgNP-preparation techniques involving the reduction of silver ions in the presence of a protective agent 
can prevent the AgNPs from aggregation and readily allow isolation of the NPs. ${ }^{18,19}$ From an economic perspective, plant extracts with various reductive groups can act as reducing and capping agents for the synthesis of NPs, which could realize more advantages over microbial synthesis in industrial production by way of cost reduction. ${ }^{14,15}$ Furthermore, natural component-mediated AgNPs utilizing reductive and pharmacological active plant extracts can integrate the advantages of bioactive components and AgNPs, ${ }^{20}$ which also displayed less toxicity against mammalian health cells. ${ }^{21}$

A number of plants are suitable for the preparation of AgNPs. For example, some reductive plants, including Aloe vera, ${ }^{22}$ Manilkara zapota, ${ }^{23}$ and Alternanthera sessilis, ${ }^{24}$ have been used for the synthesis of AgNPs with stable structure and uniform distribution. Theoretically, Agrimoniae herba contains various reductive groups, including flavonoids, phenols, and tannin, which are capable of reducing $\mathrm{Ag}^{+}$to AgNPs through specific technology. As a famous traditional herbal medicine, $A$. herba containing various active components is widely used in anticancer, ${ }^{25}$ antibacterial, ${ }^{26}$ and antiinflammatory ${ }^{27}$ treatment. Herein, the traditional herbal medicine $A$. herba extract was utilized to develop a rapid synthesis of AgNPs for in vitro antineoplastic evaluation. In this study, we investigated the influence of various reaction conditions, such as extraction time, temperature, $\mathrm{AgNO}_{3}$ concentration and $A$. herba extract amount, on the yield of $A$. herba-conjugated AgNPs (AH-AgNPs) by monitoring the conversion of $\mathrm{Ag}^{+}$ using ultraviolet (UV)-visible spectroscopy. Furthermore, we characterized the structure and morphology of AgNPs by means of transmission electron microscopy (TEM), dynamic light scattering (DLS), X-ray diffraction (XRD), and Fouriertransform infrared spectroscopy (FTIR). More importantly, the advantage of AH-AgNPs over $A$. herba extract and bare AgNPs in cytotoxicity against a human lung cancer cell line (A549 cells) was also evaluated using the 3-(4,5-dimethylthiazol-2yl)-2,5-diphenyltetra-zoliumbromide (MTT) assay.

\section{Materials and methods Materials}

A. herba was purchased from Bozhou Huqiao Pharmaceutical (Bozhou, People's Republic of China), and was authenticated by Shihui Qian, a professor at the Jiangsu Provincial Academy of Chinese Medicine. Aluminum chloride, anhydrous sodium carbonate, silver nitrate, and phosphoric acid were provided by Nanjing Chemical Reagent (purity $>99.8 \%$, analytical reagent; Nanjing, People's Republic of China). Folin-Ciocaileu reagent was obtained from Nanjing Duly Biotech (Nanjing, People's Republic of China). Rutin and gallic acid reference substances (purity $>99.5 \%$ ) were purchased from the National Institutes for Food and Drug Control (Beijing, People's Republic of China). Trisodium citrate was provided by Kunshan Jincheng Reagent (Kunshan, People's Republic of China). MTT, dimethyl sulfoxide (DMSO), Roswell Park Memorial Institute (RPMI) 1640 medium, trypsin, and phosphate-buffered saline were provided by Nanjing KeyGen Biotech (Nanjing, People's Republic of China). Fetal bovine serum (FBS) was bought from Zhejiang Tianhang Biological Technology (Hangzhou, People's Republic of China). Water in this study was provided by the Milli-Q water-purification system (EMD Millipore, Billerica, MA, USA). All other chemicals and solvents were of analytical or reagent grade.

\section{Methods}

\section{Preparation of $A$. herba extract}

Fresh $A$. herba (30 g) was culled and boiled with $300 \mathrm{~mL}$ of deionized water for 15 minutes in a $500 \mathrm{~mL}$ Erlenmeyer flask, followed by filtration in a reduced-pressure condition. At the end of this time, the volume of extract was adjusted to $300 \mathrm{~mL}$ with deionized water and stored at $4^{\circ} \mathrm{C}$ until further use.

\section{Content determination}

Total flavonoid content

In this experiment, the concentration of total flavonoid was detected at $408 \mathrm{~nm}$ by UV-visible spectroscopy using rutin as the reference substance. ${ }^{28}$ Briefly, $1 \mathrm{~mL}$ of $A$. herba extract was mixed with $2 \mathrm{~mL}$ of $1 \% \mathrm{AlCl}_{3}$ in methanol and incubated at room temperature. After coincubation for 10 minutes, UV-visible absorbance was recorded at $408 \mathrm{~nm}$, and the total flavonoid content was calculated according to a calibration curve of rutin.

\section{Total phenol content}

The total phenol content was measured at $765 \mathrm{~nm}$ by UV-visible spectroscopy using gallic acid as the reference substance. ${ }^{29}$ Briefly, $1 \mathrm{~mL}$ of Folin-Ciocaileu reagent was added dropwise into the $5 \mathrm{~mL}$ of fivefold-diluted $A$. herba extract. Subsequently, the mixture was incubated for 5 minutes at room temperature, followed by the addition of $10 \mathrm{~mL}$ of $10 \% \mathrm{Na}_{2} \mathrm{CO}_{3}$ solution. UV-visible absorbance was recorded at $765 \mathrm{~nm}$. Total phenol content was estimated by a calibration curve of gallic acid.

\section{Synthesis of AH-AgNPs}

$A$. herba extract $(10 \mathrm{~mL})$ was added to $100 \mathrm{~mL}$ of an aqueous solution of $\mathrm{AgNO}_{3}(1 \mathrm{mM})$ with vigorous stirring at room 
temperature until crimson was observed. Afterward, the reaction mixture was subjected to centrifugation at $13,000 \mathrm{rpm}$ for 15 minutes, followed by the removal of excessive silver ions through rinsing the precipitate of AH-AgNPs thrice. Finally, a crimson AH-AgNP solution was obtained when nanoparticles were redispersed in deionized water.

\section{Synthesis of bare AgNPs}

Due to the reductive ability, sodium citrate has been widely used for the preparation of AgNP systems in previous papers. $^{30,31}$ Briefly, $10 \mathrm{~mL}$ of $1 \%$ sodium citrate solution was added dropwise to $300 \mu \mathrm{g} \cdot \mathrm{mL}^{-1}$ of $\mathrm{AgNO}_{3}$ aqueous solution at $100^{\circ} \mathrm{C}$. After vigorous stirring for 10 minutes, the bare AgNPs were obtained under a $\mathrm{pH}$ environment of 4.0-6.0 using phosphoric acid as a regulator.

\section{Optimization of AH-AgNP studies}

Based on the AH-AgNP preparation, the extraction time of $A$. herba was predetermined at 2, 5, 15, 30, and 60 minutes to evaluate the influence on the yield of AH-AgNPs. Likewise, the reaction temperature was preset at $4^{\circ} \mathrm{C}, 25^{\circ} \mathrm{C}$, $40^{\circ} \mathrm{C}, 50^{\circ} \mathrm{C}$, and $60^{\circ} \mathrm{C}$ to investigate the time-dependent influence on the synthesis of AH-AgNPs. Furthermore, in order to observe the concentration-related influences on the synthesis of AH-AgNPs, various concentration gradients of $\mathrm{AgNO}_{3}$ - set at $0.1 \mathrm{mM}, 0.5 \mathrm{mM}, 1 \mathrm{mM}$, and $2 \mathrm{mM}$ - were also employed as the critical parameters. Finally, 2.5, 5, 10, 25, 50, and $75 \mathrm{~mL}$ of $A$. herba extract (equal to 0.25, 0.5, $1,2.5,5$, and $7.5 \mathrm{~g}$ crude drug, respectively) were added to $100 \mathrm{~mL}$ of $1 \mathrm{mM}$ aqueous $\mathrm{AgNO}_{3}$ solution, namely the volume ratio of $A$. herba extract to $\mathrm{AgNO}_{3}$ solution $(1 \mathrm{mM})$ varied in the range of 1:40-3:4.

The UV-visible absorption spectrum was recorded to evaluate the optimal characterization and analyze the synthesis rate of AH-AgNPs obtained under different reaction conditions at a wavelength of 300-800 nm using a UV-1800 PC UV-visible spectrophotometer (AoXi Technology Instrument, Shanghai, People's Republic of China).

\section{Characteristics of AH-AgNPs}

TEM and DLS measurements

The morphology of the optimal AH-AgNP solution was observed by TEM (Tecnai 12; Philips, Amsterdam, the Netherlands). Briefly, $15 \mu \mathrm{L}$ of the AH-AgNP solution was dropped on a carbon-coated copper grid and dried prior to measure. Ten fields of vision (including $400 \mathrm{NPs}$ in all) were selected randomly from TEM images of AH-AgNPs, and the average particle size was obtained after statistical analysis.
In addition, DLS (Nano ZS; Malvern Instruments, Malvern, UK) was also used to measure particle size and zeta potential of AH-AgNPs. Accordingly, morphologic characterization of bare AgNPs was performed as per the method described earlier.

\section{X-ray diffraction measurement}

Crystallization of the AH-AgNP dry powder obtained from freeze-drying the solution of AH-AgNPs was characterized by XRD (D8 Advance; Bruker Optik, Ettlingen, Germany) with $\mathrm{CuK} \alpha$ radiation $(\lambda=1.5406 \AA)$ and working at $40 \mathrm{kV} / 40 \mathrm{~mA}$ in the range of $10^{\circ}-80^{\circ}$ with a $2^{\circ}$-per-minute scanning rate.

\section{Fourier-transform infrared spectroscopy}

The characteristic groups of $A$. herba extract conjugated with the surface of AgNPs were characterized by FTIR (Nicolet IS10; Thermo Fisher Scientific, Waltham, MA, USA) spectroscopy in the range of $4,000-500 \mathrm{~cm}^{-1}$ at $2^{\circ} \cdot \mathrm{cm}^{-1}$ resolution. Each sample was recorded with $\mathrm{KBr}$ pellets on an FTIR spectroscope at room temperature.

\section{A549 cell line culture}

A human lung cancer cell line (A549) was obtained from KeyGen Biotech. The cells were cultured in RPMI 1640 medium supplemented with $10 \%$ FBS, $1 \%$ nonessential amino acids, $100 \mathrm{U} \cdot \mathrm{mL}^{-1}$ streptomycin, and $100 \mu \mathrm{g} \cdot \mathrm{mL}^{-1}$ penicillin at $37^{\circ} \mathrm{C}$ under an atmosphere of $5 \% \mathrm{CO}_{2}$ and $90 \%$ relative humidity. The cells were subcultivated every 3-4 days (at 70\% confluence) using trypsin at a split ratio of 1:4.

\section{In vitro cytotoxicity studies}

A549 cells were used to investigate the cytotoxicity of AH-AgNPs in vitro by MTT assay. The A549 cells were seeded into 96 -well culture plates at a density of $5 \times 10^{3}$ cells per well. After $80 \%$ confluence, the culture medium was removed, and $200 \mu \mathrm{L}$ of each solution $(100 \mu \mathrm{L}$ of FBS-free culture medium with $100 \mu \mathrm{L}$ of AH-AgNPs, bare AgNPs, and $A$. herba solution at various concentrations) and a negative control $(100 \mu \mathrm{L}$ of FBS-free culture medium with $100 \mu \mathrm{L}$ of culture medium) were added to the wells. After further cell culture for 24 hours, $20 \mu \mathrm{L}$ of sterile MTT solution $\left(5 \mathrm{mg} \cdot \mathrm{mL}^{-1}\right)$ was added to each well, and then the cells were cultured for a further 4 hours at $37^{\circ} \mathrm{C}$. Afterward, the culture medium was removed, and $100 \mu \mathrm{L}$ of DMSO was added to dissolve the produced formazan crystals. The optical density (OD) at $490 \mathrm{~nm}$ was measured by using a Multiskan MK3 microplate reader (Thermo Fisher Scientific). Cell viability (\%) was calculated according to the following 
formula: cell viability $(\%)=(\mathrm{OD}$ of test group/OD of control group) $\times 100 \%$. The drug concentration causing $50 \%$ cellgrowth inhibition $\left(\mathrm{IC}_{50}\right)$ was calculated using SPSS 17.0 (SPSS, Inc., Chicago, IL, USA) software.

\section{Statistical analysis}

All experiments were conducted in triplicate unless otherwise specified. Statistical analysis was performed with SPSS 17.0 software. All the results were expressed as means plus or minus standard deviation. Student's $t$-test was used to evaluate the differences between groups. A value of $* * P<0.01$ was considered extremely significant.

\section{Results}

\section{Content determination}

$A$. herba is known to be rich in diverse bioactive components (such as flavonoids and phenols) that might play a significant role in the bioreduction of AgNPs. Phytochemical analysis of $A$. herba extract $\left(0.1 \mathrm{~g}\right.$ crude $\left.\mathrm{drug} \cdot \mathrm{mL}^{-1}\right)$ revealed a high level of total flavonoid content up to $4.0 \mathrm{mg} \cdot \mathrm{mL}^{-1}$. Likewise, A. herba extract $\left(0.1 \mathrm{~g}\right.$ crude $\left.\operatorname{drug} \cdot \mathrm{mL}^{-1}\right)$ was found to be rich in total phenols $\left(2.6 \mathrm{mg} \cdot \mathrm{mL}^{-1}\right)$. Accordingly, the high level of total flavonoids and phenols showed the potential capability of reducing $\mathrm{Ag}^{+}$to $\mathrm{AgNPs}$.

\section{Effect of extraction time of $A$. herba on the synthesis of AH-AgNPs}

The absorption spectrum of AH-AgNPs was recorded at different extracted times of $A$. herba (Figure 1).

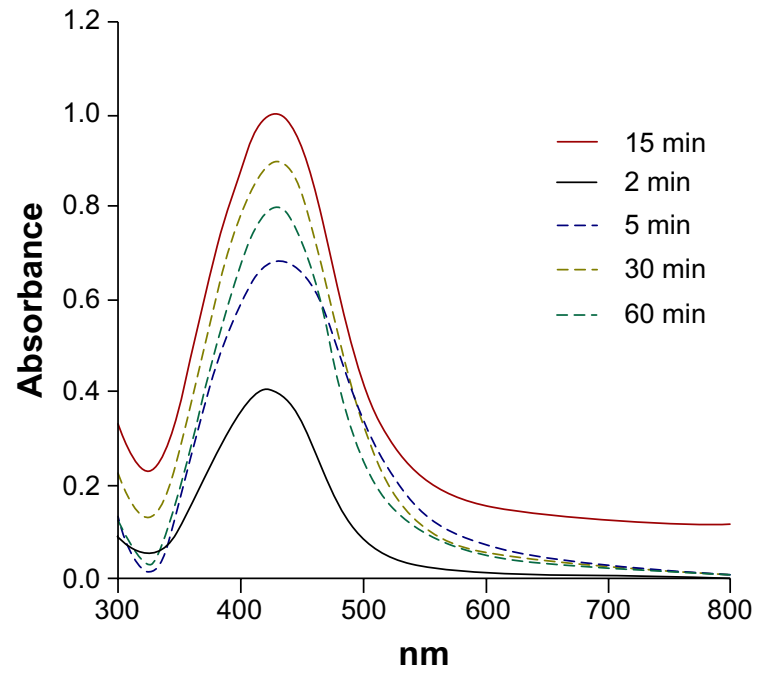

Figure I Ultraviolet-visible spectra of AH-AgNPs obtained via Agrimoniae herba extract and I $\mathrm{mM} \mathrm{AgNO}_{3}$ for various extraction times.

Notes: The full line represents the optimum reaction time, and the dashed lines represent assayed reaction conditions. The ordinate represents the maximal ultraviolet absorption within a range of $300-800 \mathrm{~nm}$.

Abbreviation: $\mathrm{AH}-\mathrm{AgNPs}$, Agrimoniae herba-conjugated $\mathrm{Ag}$ nanoparticles.
The maximum amount of $A$. herba conjugated with AgNPs was found after extraction for 15 minutes. Interestingly, a longer extraction time had a comparatively lower yield. Consequently, the extraction time played a key role in the synthesis of AH-AgNPs, the main reason might be connected with different extraction time due to various active components.

\section{Effect of reaction temperature and time on the synthesis of $\mathrm{AH}-\mathrm{AgNPs}$}

We further prearranged various temperatures from $4^{\circ} \mathrm{C}$ to $60^{\circ} \mathrm{C}$ and different reaction times to observe the peak wavelength of AH-AgNPs and investigate the influence of temperature and reaction time on the yield of AH-AgNPs. As shown in Figure 2, an expansion of reaction time was accompanied with an obvious improvement in the yield of AH-AgNPs before 8 hours, but no significant enhancement was found after 8 hours. Also, temperatures below or above $25^{\circ} \mathrm{C}$ were unable obtain the maximal synthesis rate of AH-AgNPs.

\section{Effect of Ag ion concentration on the synthesis of AgNPs}

$\mathrm{Ag}^{+}$bioreduction mediated by $A$. herba extract was also monitored by recording absorption spectra at different $\mathrm{AgNO}_{3}$ concentrations. As shown in Figure 3, the absorbance at 447

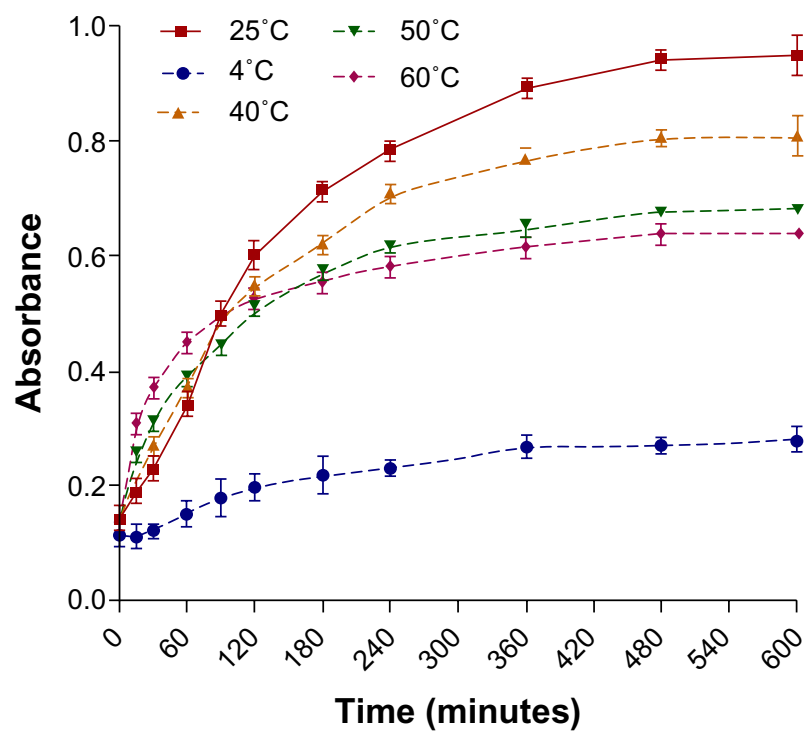

Figure 2 Time course of $\mathrm{AH}-\mathrm{AgNPs}$ prepared via Agrimoniae herba extract and I $\mathrm{mM} \mathrm{AgNO}_{3}$ under different reaction temperatures.

Notes: The full line represents the optimum reaction temperature, and the dashed lines represent assayed reaction temperatures. Data represent means \pm standard deviation. The ordinate represents the maximal ultraviolet absorption within a range of 300-800 nm.

Abbreviation: AH-AgNPs, Agrimoniae herba-conjugated Ag nanoparticles. 


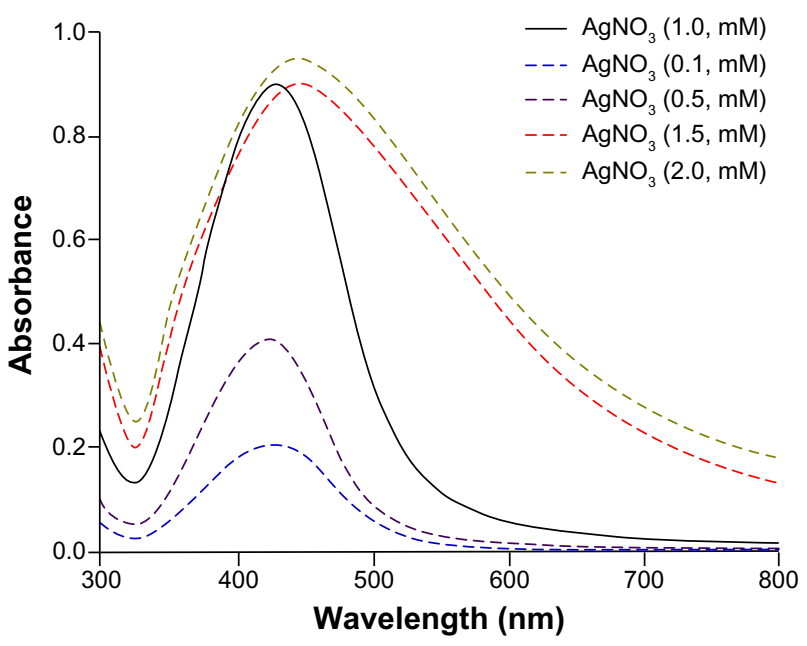

Figure 3 Ultraviolet-visible spectra of $\mathrm{AH}-\mathrm{AgNPs}$ obtained via Agrimoniae herba extract at various concentrations of $\mathrm{AgNO}_{3}$.

Notes: The full line represents the optimum concentration of $\mathrm{AgNO}_{3}$, and the dashed lines represent assayed concentrations of $\mathrm{AgNO}_{3}$. The ordinate represents the maximal ultraviolet absorption within a range of 300-800 nm.

Abbreviation: $\mathrm{AH}-\mathrm{AgNPs}$, Agrimoniae herba-conjugated Ag nanoparticles.

$\mathrm{nm}$ increased obviously as the concentration increased from 0.1 to $1.0 \mathrm{mM}$. However, the absorption wavelength was slightly shifted when $\mathrm{AgNO}_{3}$ concentrations were higher than $1.0 \mathrm{mM}$, at which the comparative agglomeration was also speculated to be due to the broadening of peak width at half height. ${ }^{32}$

\section{Effect of A. herba extract amount on the synthesis of AH-AgNPs}

Optimization of the amount of $A$. herba extract was carried out by recording UV-visible absorption of AH-AgNPs. In this experiment, the reaction was monitored by varying ratios of extract solution to silver nitrate $(1: 40,1: 20,1: 10,1: 4,1: 2$, and $3: 4, v / v)$. As shown in Figure 4, the maximal absorbance (represented yield) was displayed when the ratio of extract to silver nitrate was predetermined at 1:10 (v/v). According to the results of visual observation, with the amount of $A$. herba extract increased, consequent changes in the color of AH-AgNPs were observed from yellow to reddish-yellow, and further crimson.

\section{Stability of AH-AgNPs}

In order to evaluate the stability of the AH-AgNPs in an aqueous system, the absorption spectrum of each optimized sample was detected after storage at room temperature for 2 months. As shown in Figure 5, the absorption peak of AH-AgNPs shifted slightly from 445 to $450 \mathrm{~nm}$, but no significant changes in peak intensity were observed. This suggested that AgNPs mediated by $A$. herba extract prepared

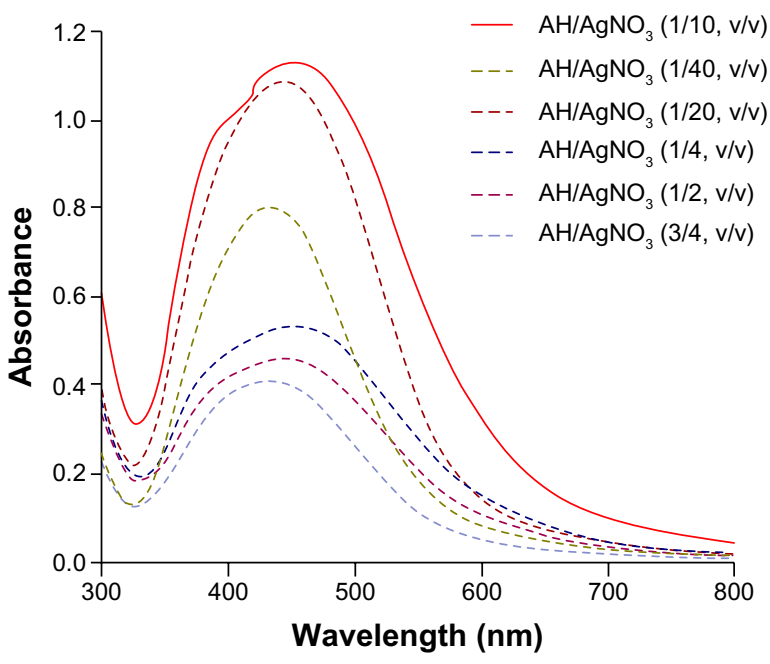

Figure 4 Ultraviolet-visible spectra of $\mathrm{AH}-\mathrm{AgNPs}$ obtained at various volume ratios of Agrimoniae herba extract to $\mathrm{AgNO}_{3}$.

Notes: The full line represents the optimum amount of $A$. herba extract, and the dashed lines represent the assayed amount of $A$. herba extract. The ordinate represents the maximal ultraviolet absorption within a range of $300-800 \mathrm{~nm}$. Abbreviation: $\mathrm{AH}-\mathrm{AgNPs}$, Agrimoniae herba-conjugated $\mathrm{Ag}$ nanoparticles.

under optimum condition might have acceptable stability even after 60 days.

\section{TEM and DLS analysis of AH-AgNPs}

In order to investigate the microstructure of AH-AgNPs and evaluate the relevant preparation technology, AH-AgNP morphology was assessed by TEM. As shown in Figure 6A, well-defined spherical particles with homogeneous distributional size were observed clearly, which confirmed that the AH-AgNPs were successfully prepared. Meanwhile, the average particle size of $\sim 11 \mathrm{~nm}$ was calculated after statistical analysis (see Figure 6B). On the other hand, small size

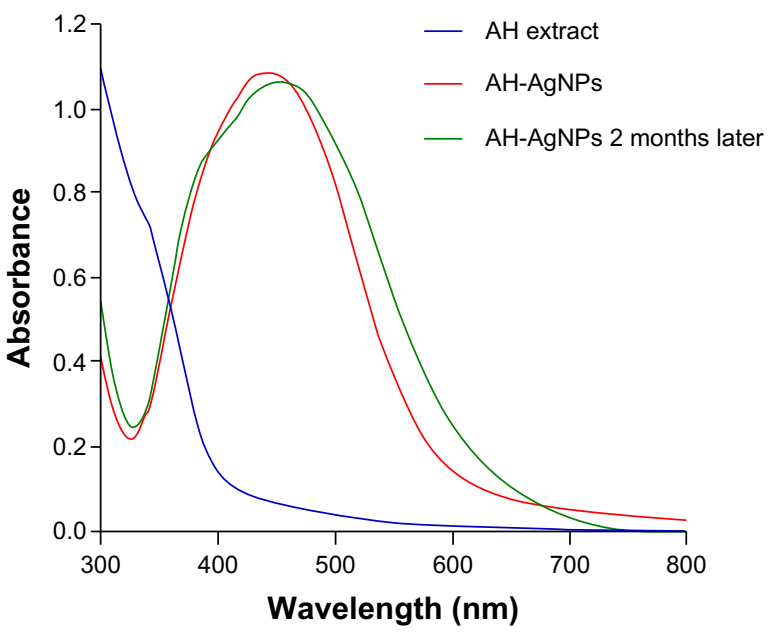

Figure 5 Evaluation of stability of Agrimoniae herba extract, AH-AgNPs, and $\mathrm{AH}-\mathrm{AgNPs} 2$ months later by using an ultraviolet-visible instrument.

Abbreviation: AH-AgNPs, Agrimoniae herba-conjugated Ag nanoparticles. 

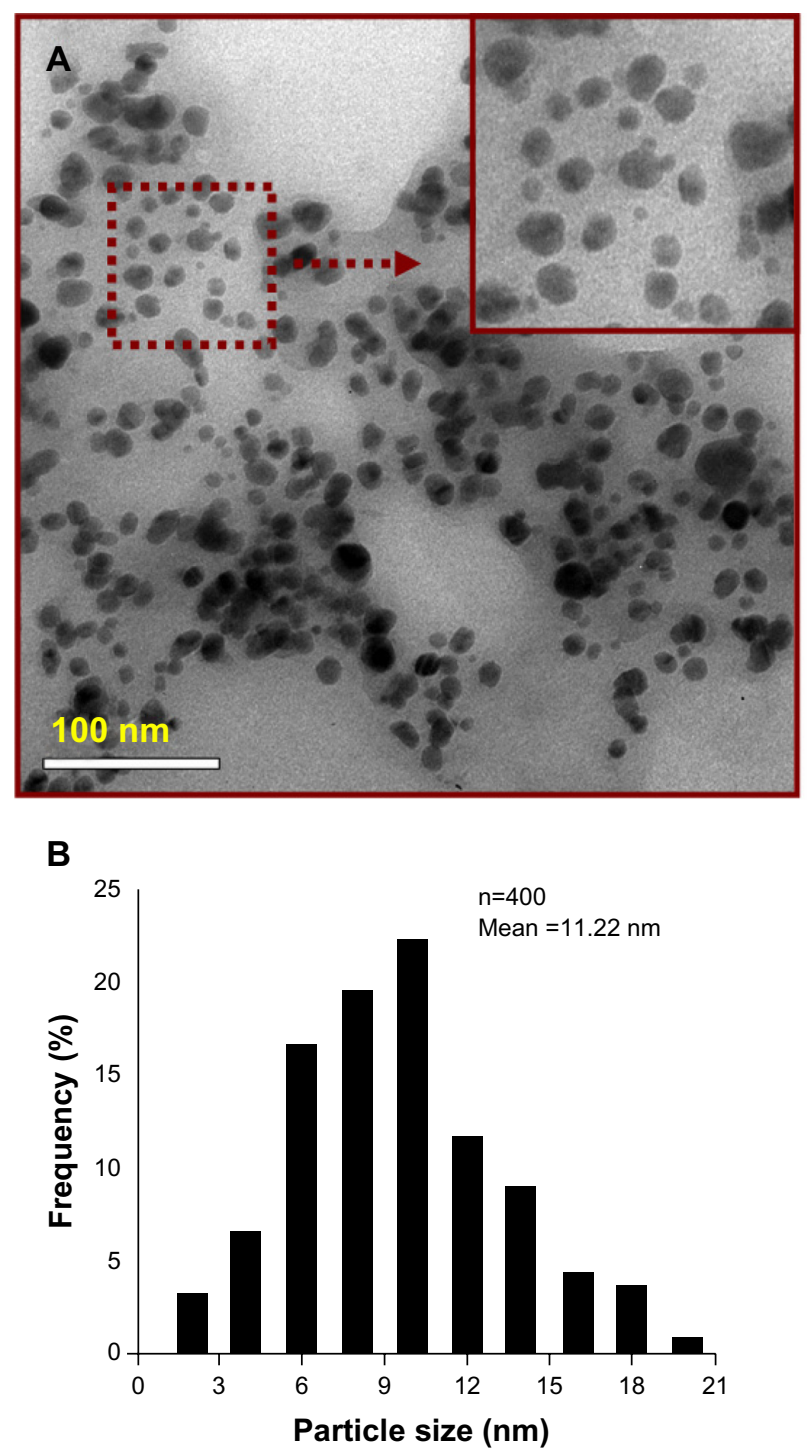

Figure 6 (A and B) Morphologic characteristics. (A) TEM image and (B) particlesize distribution of $\mathrm{AH}-\mathrm{AgNPs}$ synthesized via Agrimoniae herba extract and $\mathrm{AgNO}_{3}$. Abbreviations: TEM, transmission electron microscopy; AH-AgNPs, Agrimoniae herba-conjugated Ag nanoparticles.

$(30.34 \pm 5.9 \mathrm{~nm})$, narrow polydispersity index $(0.142)$, and highly negative zeta potential $(-36.8 \pm 2.5 \mathrm{mV})$ were also measured by DLS (see Figure 7). This suggested a superior and highly efficient preparation technology of AH-AgNPs and a stable system contributed to by highly negative potential. In addition, the morphology of bare AgNPs also showed similar results for size $(26.42 \pm 3.2 \mathrm{~nm})$ and zeta potential $(-28.3 \pm 4.7 \mathrm{mV}$, Figures S1 and S2).

\section{Powder X-ray diffraction analysis}

The crystalline nature of AgNPs was confirmed by XRD analysis (Figure 8). XRD patterns showed four distinct diffraction peaks at $38.52^{\circ}, 44.30^{\circ}, 64.23^{\circ}$, and $77.56^{\circ}$, which can be attributed to the (111), (200), (220), and (311) crystallographic planes of the face-centered cubic silver crystals. ${ }^{33,34}$ The broadening of Bragg's peaks indicated the formation of AgNPs, which conformed to the database of the Joint Committee on Powder Diffraction Standards file 04-0783. Average size was calculated using the DebyeScherrer equation by determining the width of the Bragg's reflection:

$$
\mathrm{D}=\mathrm{K} \lambda / \beta \cos \theta,
$$

where D represents the mean crystal size of NPs, K represents the Scherer coefficient (0.89), $\lambda$ is the wavelength of the $\mathrm{X}$-ray radiation source (1.5406 $\AA$ ), $\beta$ represents the width of the XRD peak at half height, and $\theta$ represents the Bragg angle. According to the Debye-Scherrer equation, the average crystallite size of AH-AgNPs was calculated to be about $11.53 \mathrm{~nm}$ (Table 1), which was in line with the result offered by TEM.

\section{FTIR spectroscopy analysis}

With the purpose of identifying the characteristic groups of $A$. herba extract and AH-AgNP freeze-dried powder, the FTIR absorption spectrum of each sample is displayed in Figure 9. Compared with $A$. herba extract, obvious weakness in characteristic peaks at $1,360 \mathrm{~cm}^{-1}$ and $3,230 \mathrm{~cm}^{-1}$, which were assigned to the stretching and bending vibrations of hydroxyl groups, was observed from the FTIR spectra of AHAgNPs, suggesting that the consumption of hydroxyl groups was responsible for the reduction of $\mathrm{Ag}^{+} .{ }^{35,36}$ The vibrational bands corresponding to the bonds were derived from watersoluble extracts, such as flavonoids and polyphenols present in $A$. herba ${ }^{21,37}$ In addition, the peaks at $2,918.77 \mathrm{~cm}^{-1}$, $1,600.81 \mathrm{~cm}^{-1}$, and $1,021.10 \mathrm{~cm}^{-1}$ can be attributed to the stretching vibration of the $-\mathrm{N}-\mathrm{H}-,-\mathrm{C}=\mathrm{C}-$, and $-\mathrm{C}-\mathrm{O}-\mathrm{C}$ groups, respectively. ${ }^{1,38-40} \mathrm{On}$ the basis of these results, it is suggested that the AgNPs were coated with reductive components mentioned earlier, and hence could feasibly inhibit further aggregation in an aqueous environment.

\section{Cytotoxicity of AH-AgNPs against $A 549$ cells}

In order to confirm the advantage of AH-AgNPs over $A$. herba extract in antitumor effects in vitro, A549 cell-viability assays were performed by conventional MTT. As shown in Figure 10, AH-AgNPs had a predominant cytotoxic effect against A549 cells, suggesting a significant enhancement on in vitro antitumor efficacy compared with $A$. herba extract. Also, bare AgNP cytotoxicity evaluation allowed us to further 
A

Size distribution by intensity

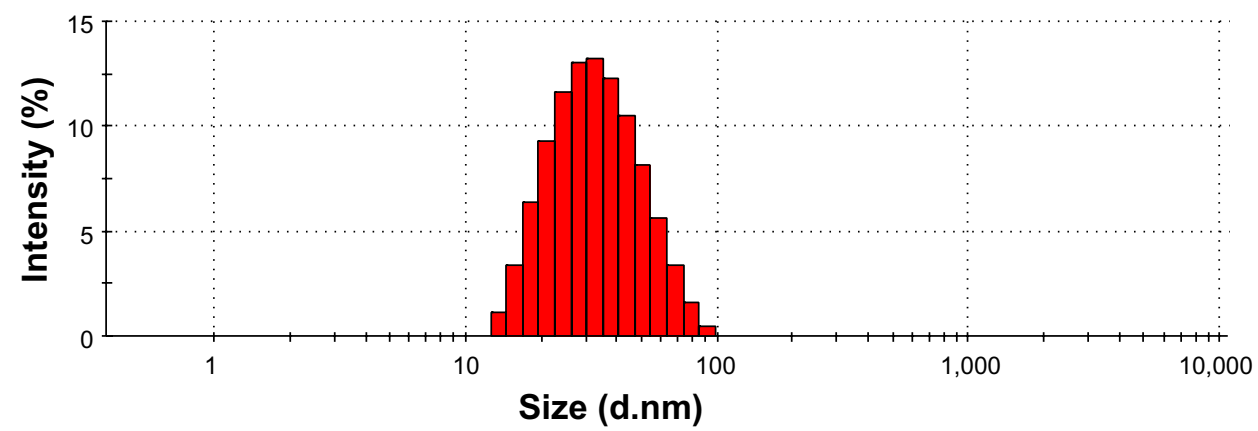

B Zeta-potential distribution

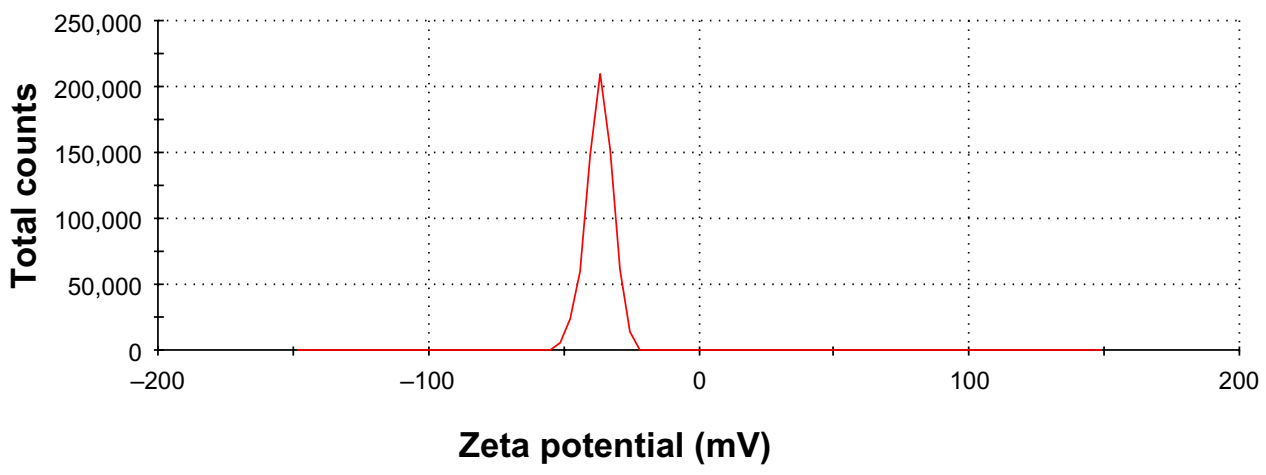

Figure 7 (A and B) Structure characteristics by DLS patterns. (A) Size distribution of AH-AgNPs; (B) zeta-potential analysis of AH-AgNPs. Abbreviations: DLS, dynamic light scattering; AH-AgNPs, Agrimoniae herba-conjugated Ag nanoparticles.

define the feasibility of AH-AgNPs in antitumor therapy. The $\mathrm{IC}_{50}$ values of AH-AgNPs, bare AgNPs, and $A$. herba extract after coincubation with A549 cells for 24 hours were 38.13, 184.87 , and $1.147 \times 10^{4} \mu \mathrm{g} \cdot \mathrm{mL}^{-1}$, respectively.

\section{Discussion}

Malignant tumors are fatal diseases that threaten human health. Therefore, tremendous efforts have been put into

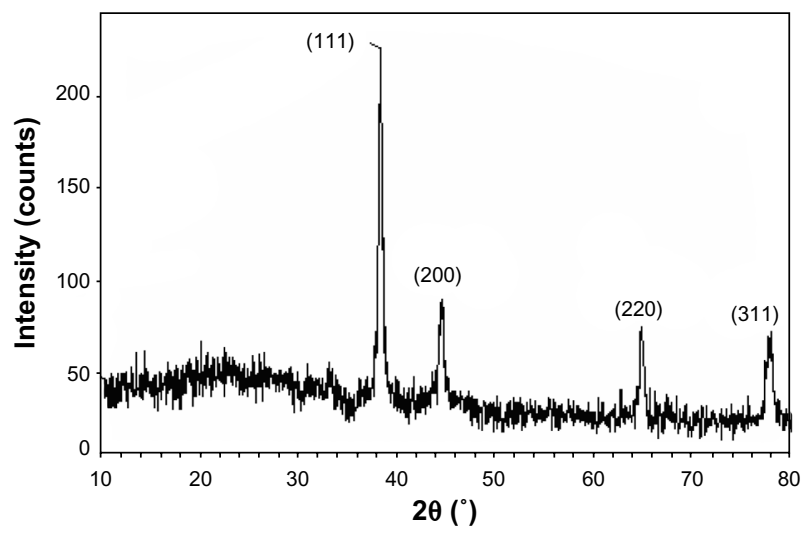

Figure 8 X-ray diffraction patterns of $\mathrm{AH}-\mathrm{AgNPs}$. Abbreviation: AH-AgNPs, Agrimoniae herba-conjugated Ag nanoparticles. exploring effective therapeutic modalities for antitumor treatment. As two approved effective anticancer media, $A$. herba and AgNPs have been applied in the clinical setting recently. ${ }^{41}$ In this study, AH-AgNPs were rapidly synthesized for the first time, and their more potent antineoplastic effect in vitro was confirmed compared with that of $A$. herba extract and bare AgNPs.

$A$. herba extract containing rich flavonoids, phenols and tannin played a key role in the process for bioreduction of $\mathrm{Ag}^{+}$to AgNPs. ${ }^{42}$ Preparation-optimization studies showed that the yield of AH-AgNPs decreased when the extraction time was longer than 15 minutes, probably because the reductive

Table I X-ray diffraction measurement of AH-AgNPs for evaluating the average crystallite size

\begin{tabular}{lllll}
\hline (hkl) & $\mathbf{2 \theta}(\mathbf{d e g})$ & $\boldsymbol{\theta}(\mathbf{d e g})$ & $\boldsymbol{\beta}(\mathrm{rad})$ & $\mathbf{D}(\mathbf{n m})$ \\
\hline$(\mathrm{III})$ & 38.52 & 19.26 & 0.0133 & 10.96 \\
$(200)$ & 44.30 & 22.15 & 0.0131 & 11.23 \\
$(220)$ & 64.23 & 32.16 & 0.0137 & 11.78 \\
$(3 \mathrm{II})$ & 77.56 & 38.78 & 0.0144 & 12.17 \\
\hline
\end{tabular}

Notes: $D$ represents the mean crystal size of AH-AgNPs, $\beta$ represents the width of the XRD peak at half height, and $\theta$ represents the Bragg angle.

Abbreviations: $\mathrm{AH}-\mathrm{AgNPs}$, Agrimoniae herba-conjugated $\mathrm{Ag}$ nanoparticles; $\mathrm{XRD}$, X-ray diffraction. 


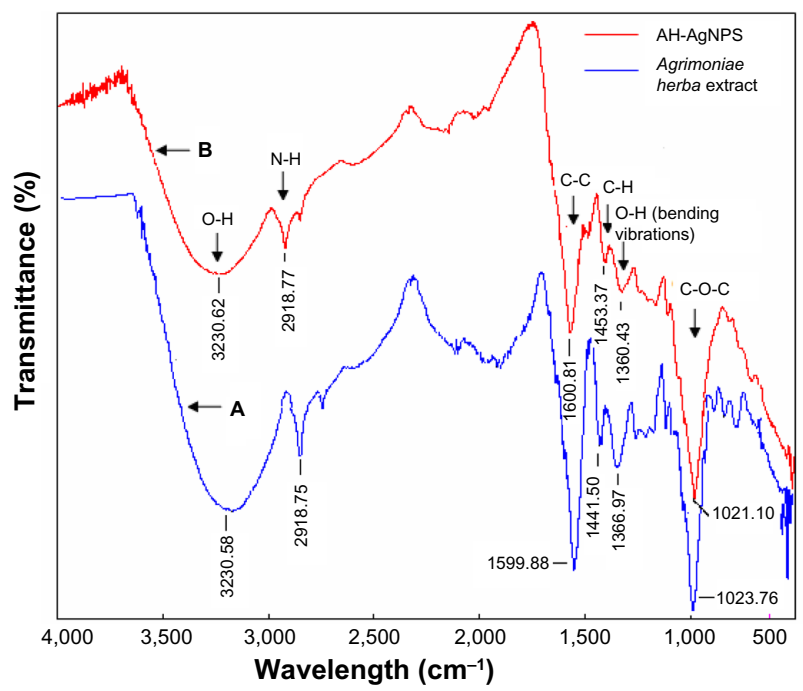

Figure 9 Fourier-transform infrared absorption spectra of Agrimoniae herba extract (A), and (B) AH-AgNP freeze-dried power.

Abbreviation: $\mathrm{AH}-\mathrm{AgNPs}$, Agrimoniae herba-conjugated $\mathrm{Ag}$ nanoparticles.

compositions were destroyed in $A$. herba extract after enduring boiling for a long time. Likewise, the synthesis rate of $\mathrm{AH}-\mathrm{AgNPs}$ decreased when the temperature was higher than $25^{\circ} \mathrm{C}$ in the process of preparation. Without good dispersion or lack of $A$. herba extract as a stabilizing agent, AgNPs were easily agglomerated. To some extent, $A$. herba extract also played a role in the stabilization of AH-AgNPs. On the other hand, particle size, polydispersity index, and zeta potential were important characteristic parameters for testing the long-term storage stability and structure characterization of a drug-delivery system. In DLS measurements, AH-AgNPs were dissolved in deionized water and water molecules encapsulated in silver nanoparticles surface, leading to the different sizes between DLS and TEM measurements. According to a previous paper, the development of crimson color in AH-AgNP solution might be attributed to the occurrence of surface-plasmon resonance, further identifying the successful preparation of AH-AgNPs. ${ }^{35}$ According to the results of FTIR and DLS, the advantages of optimum AHAgNPs, such as accepted stability and uniform dispersion, were attributed to $A$. herba extract conjugation with $\mathrm{Ag}^{+}$. With regard to synthetic rate, $A$. herba-mediated reduction of $\mathrm{Ag}^{+}$was completed within 8 hours, which was about threefold improved in comparison with some other plants, such as Curcuma longa ${ }^{38}$ which needed 24 hours for complete synthesis of AgNPs. The obtained results indicated a rapid, economical, and effective preparation.

In this study, the strengths of AH-AgNPs over A. herba extract and bare AgNPs in cytotoxicity was evaluated by MTT assay. On the basis of $\mathrm{IC}_{50}$ of AH-AgNPs and bare

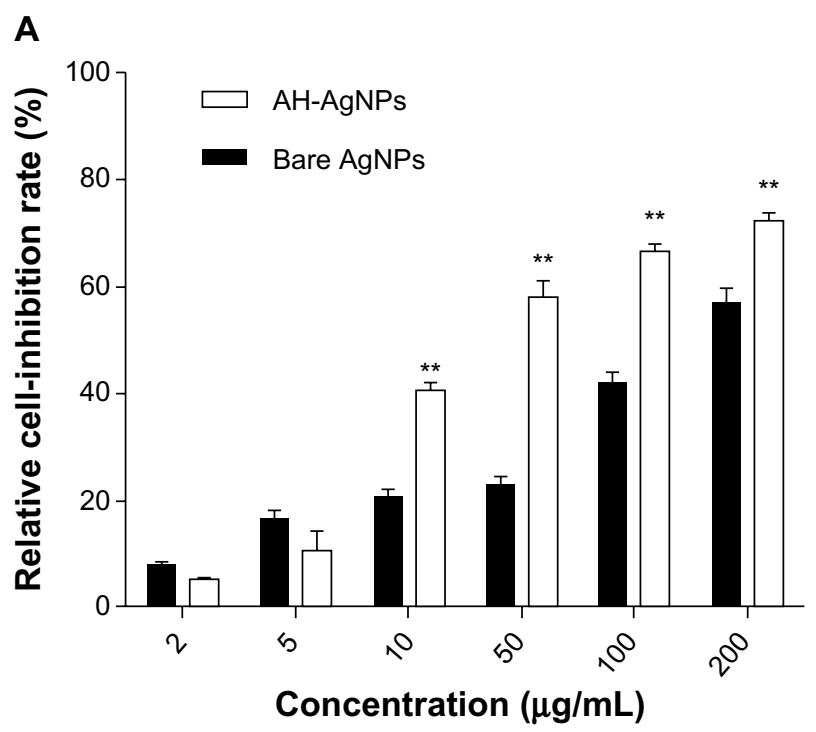

B

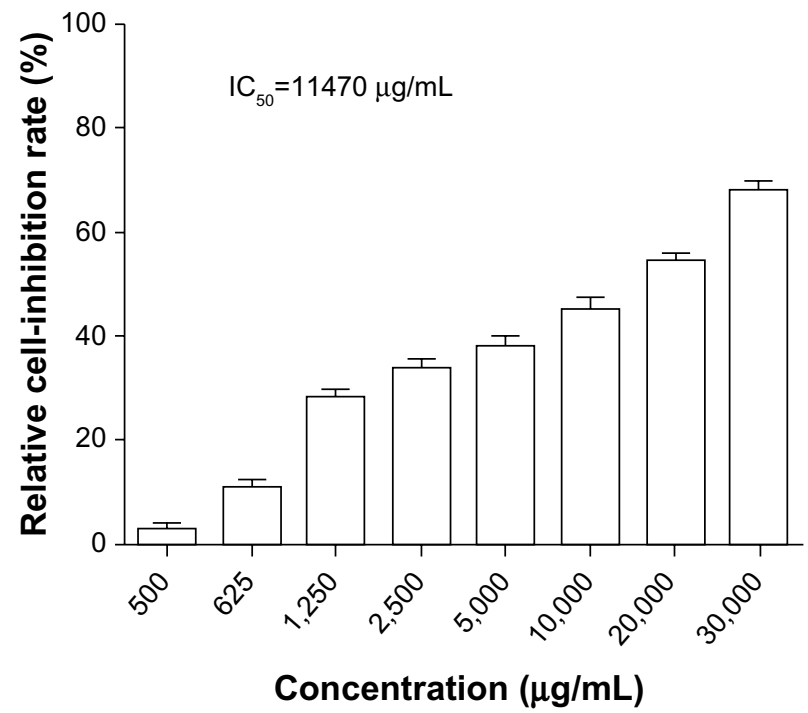

Figure 10 Cytotoxicity of (A) AH-AgNPs and bare-AgNPs, and (B) Agrimoniae Herba extract against A549 cells.

Notes: ${ }^{* *} P<0.0$ I versus bare AgNPs. Data represent means \pm standard deviation. Abbreviations: AH-AgNPs, Agrimoniae herba-conjugated Ag nanoparticles; $\mathrm{IC}_{50}$, half-maximal inhibitory concentration.

AgNPs, AgNPs with or without $A$. herba extract exhibited evident cytotoxicity against A549 cells, suggesting that AgNPs, as a drug carrier, also can act as an effective anticancer agent. Furthermore, it was reasonable that the cell-growth inhibition of AH-AgNPs was about fivefold higher than that of bare AgNPs, which demonstrated that the antitumor potential of AH-AgNPs was more promising than that of bare AgNPs. The noteworthy cytotoxicity of $\mathrm{AH}-\mathrm{AgNPs}$ further indicated a clear conjugation between A. herba extract and the surface of AgNPs. The mechanism of cell death by AH-AgNPs in A549 cells will need to be researched in the future. 


\section{Conclusion}

In summary, $A$. herba-conjugated AgNPs were synthesized using a green and highly effective preparation technology. This synthesis approach has potential in the industrial process, and offers an alternative to traditional physical and chemical methods. More importantly, the cytotoxicity of AH-AgNPs against A549 cells had an advantage over that of bare AgNPs and $A$. herba extract. In conclusion, this study suggests that AH-AgNPs exhibit the potential to be a promising drug-delivery system in antitumor therapy.

\section{Acknowledgment}

This work was financially supported by the Jiangsu Provincial Chinese Medicine Leading Talent Project (LJ200913).

\section{Disclosure}

The authors report no conflicts of interest in this work.

\section{References}

1. Oberdörster G, Maynard A, Donaldson K, et al. Principles for characterizing the potential human health effects from exposure to nanomaterials: elements of a screening strategy. Part Fibre Toxicol. 2005;2:8.

2. Gittins DI, Bethell D, Schiffrin DJ, Nichols RJ. A nanometre-scale electronic switch consisting of a metal cluster and redox-addressable groups. Nature. 2000;408(6808):67-69.

3. Darroudi M, Ahmad MB, Abdullah AH, Ibrahim NA. Green synthesis and characterization of gelatin-based and sugar-reduced silver nanoparticles. Int J Nanomedicine. 2011;6:569-574.

4. Xu J, Xiao X, Ren F, et al. Enhanced photocatalysis by coupling of anatase $\mathrm{TiO}_{2}$ film to triangular Ag nanoparticle island. Nanoscale Res Lett. 2012;7(1):239.

5. Neu HC. The crisis in antibiotic resistance. Science. 1992;257(5073): 1064-1073.

6. Pitout JD, Sanders CC, Sanders WE Jr. Antimicrobial resistance with focus on beta-lactam resistance in Gram-negative bacilli. Am J Med. 1997;103(1):51-59.

7. Craig WA. Antimicrobial resistance issues of the future. Diagn Microbiol Infect Dis. 1996;25(4):213-217.

8. Shakibaie MR, Kapadnis BP, Dhakephalker P, Chopade BA. Removal of silver from photographic wastewater effluent using Acinetobacter baumannii BL54. Can J Microbiol. 1999;45(12):995-1000.

9. Patwardhan RB, Dhakephalkar PK, Niphadkar KB, Chopade BA. Incidence and prevalence of nosocomial pathogens in ICU with special reference to multiresistant Acinetobacter baumannii harboring multiple plasmids. Indian J Med Res. 2008;128(8):178-187.

10. Chen X, Schluesener HJ. Nanosilver: a nanoproduct in medical application. Toxicol Lett. 2008;1(4):1-12.

11. He QG, Liu J, Huang CW, Wu W. Synthesis and characterization of a silver incorporated magnetic nanocomposite with enhanced antibacterial activity. Sci Adv Mater. 2014;6(1):366-376.

12. He Q, Huang C, Liu J. Preparation, characterization and antibacterial activity of magnetic greigite and $\mathrm{Fe}_{3} \mathrm{~S}_{4} / \mathrm{Ag}$ nanoparticles. Nanosci Nanotechnol Lett. 2014;6(1):10-17.

13. Li Y, Leung P, Yao L, Song QW, Newton E. Antimicrobial effect of surgical masks coated with nanoparticles. J Hosp Infect. 2006;62(1):58-63.

14. Strohal R, Schelling M, Takacs M, Jurecka W, Gruber U, Offner F. Nanocrystalline silver dressings as an efficient anti-MRSA barrier: a new solution to an increasing problem. J Hosp Infect. 2005;60(3): 226-230.
15. Zhang WZ, Qiao XL, Chen JG. Synthesis of silver nanoparticles effects of concerned parameters in water/oil microemulsion. Mater Sci Eng B. 2007;142(1):1-15.

16. Dai ZG, Xiao XH, Zhang YP, et al. In situ Raman scattering study on a controllable plasmon-driven surface catalysis reaction on $\mathrm{Ag}$ nanoparticle arrays. Nanotechnology. 2012;23(33):335701.

17. Mandal D, Bolander ME, Mukhopadhyay D, Sarkar G, Mukherjee P. The use of microorganisms for the formation of metal nanoparticles and their application. Appl Microbiol Biotechnol. 2006;69(5):485-492.

18. Quiros I, Yamada M, Kubo K, Mizutani J, Kurihara M, Nishihara H. Preparation of alkanethiolate-protected palladium nanoparticles and their size dependence on synthetic conditions. Langmuir. 2002;18(4): 1413-1418.

19. Chen S, Huang K, Stearns JA. Alkanethiolate-protected palladium nanoparticles. Chem Mater. 2000;12:540-541.

20. Gengan RM, Anand K, Phulukdaree A, Chuturgoon A. A549 lung cell line activity of biosynthesized silver nanoparticles using Albizia adianthifolia leaf. Colloids Surf B Biointerfaces. 2013;105:87-91.

21. Lansdown AB. Silver in health care: antimicrobial effects and safety in use. Curr Probl Dermatol. 2006;33:17-34.

22. Chandran SP, Chaudhary M, Pasricha R, Ahmad A, Sastry M. Synthesis of gold nanoparticles and silver nanoparticles using Aloe vera plant extract. Biotechnol Prog. 2006;22(2):577-583.

23. Kamaraj C, Rajakumar G, Rahuman AA, et al. Feeding deterrent activity of synthesized silver nanoparticles using Manilkara zapota leaf extract against the house fly, Musca domestica (Diptera: Muscidae). Parasitol Res. 2012;111(6):2439-2448.

24. Niraimathi KL, Sudha V, Lavanya R, Brindha P. Biosynthesis of silver nanoparticles using Alternanthera sessilis (Linn.) extract and their antimicrobial, antioxidant activities. Colloids Surf B Biointerfaces. 2013;102:288-291.

25. Li WY, Chan SW, Guo DJ, Yu HF. Correlation between antioxidative power and anticancer activity in herbs from traditional Chinese medicine formulae with anticancer therapeutic effect. Pharm Biol. 2007;45(7): 541-546.

26. Park NY, Park KN, Lee SH. Antimicrobial activities and food preservative effects of Agrimoniae herba. J Korean Soc Food Sci Nutr. 2004;33(2):244-248.

27. Gong CG, Zhang GQ, Wang XY, Lei YH. Experimental studies of antiinflammatory and analgesic effects of extract from Herba agrimoniae [sic]. J Pharm Pract. 2006;2006(6):339-341, 356.

28. Jiang P, Burczynski FC, Campbell C, Pierce G, Austria JA, Briggs CJ. Rutin and flavonoid contents in three buckwheat species Fagopyrum esculentum, F. tataricum, and F. homotropicum and their protective effects against lipid peroxidation. Food Res Int. 2007;40(3): 356-364.

29. Ainsworth EA, Gillespie KM. Estimation of total phenolic content and other oxidation substrates in plant tissues using Folin-Ciocalteu reagent. Nat Protoc. 2007;2(4):875-877.

30. Link S, Wang ZL, El-Sayed MA. Alloy formation of gold-silver nanoparticles and the dependence of the plasmon absorption on their composition. J Phys Chem B. 1999;103(18):3529-3533.

31. Wei Y, Jin RC, Chad A. Mirkin DNA-modified core-shell Ag/Au nanoparticles. J Am Chem Soc. 2001;123(32):7961-7962.

32. Jha AK, Prasad K, Kumar V, Prasad K. Biosynthesis of silver nanoparticles using Eclipta leaf. Biotechnol Prog. 2009;25(5): 1476-1479.

33. Ahmad MB, Shameli K, Darroudi M, Yunus WM, Ibrahim NA. Synthesis and characterization of silver/clay nanocomposites by chemical reduction method. Am J Appl Sci. 2009;6(11):1909-1914.

34. Borchert H, Shevchenko EV, Robert A, Kornowski A, Haase M, Weller H Determination of nanocrystal sizes: a comparison of TEM, SAXS, and XRD studies of highly monodisperse $\mathrm{CoPt}_{3}$ particles. Langmuir. 2005;21(5):1931-1936.

35. Elavazhagan T, Arunachalam KD. Memecylon edule leaf extract mediated green synthesis of silver and gold nanoparticles. Int $J$ Nanomedicine. 2011;6:1265-1278. 
36. Geethalakshmi R, Sarada DVL. Gold and silver nanoparticles from Trianthema decandra: synthesis, characterization, and antimicrobial properties. Int J Nanomedicine. 2012;7:5375-5384.

37. Philip D, Unni C. Extracellular biosynthesis of gold and silver nanoparticles using Krishna tulsi (Ocimum sanctum) leaf. Physica E Low Dimens Syst Nanostruct. 2011;43(7):1318-1322.

38. Shameli K, Ahmad MB, Zamanian A, et al. Green biosynthesis of silver nanoparticles using Curcuma longa tuber powder. Int J Nanomedicine. 2012;7:5603-5610.

39. Shaligram NS, Bule M, Bhambure R, et al. Biosynthesis of silver nanoparticles using aqueous extract from the compactin producing fungal strain. Process Biochem. 2009;44(8):939-943.
40. Valli JS, Vaseeharan B. Biosynthesis of silver nanoparticles by Cissusquadrangularis extracts. Mater Lett. 2012;82:171-173.

41. Kim SH, Lee TH, Yang DC. Shiquandabutangjiaweibang inhibits tumor metastasis and angiogenesis via regulation of topoisomerase-1. J Ethnopharmacol. 2005;98(1-2):157-162.

42. Egorova EM, Revina AA. Synthesis of metallic nanoparticles in reverse micelles in the presence of quercetin. Colloids Surf A Physicochem Eng Asp. 2000;168(1):87-96. 


\section{Supplementary materials}

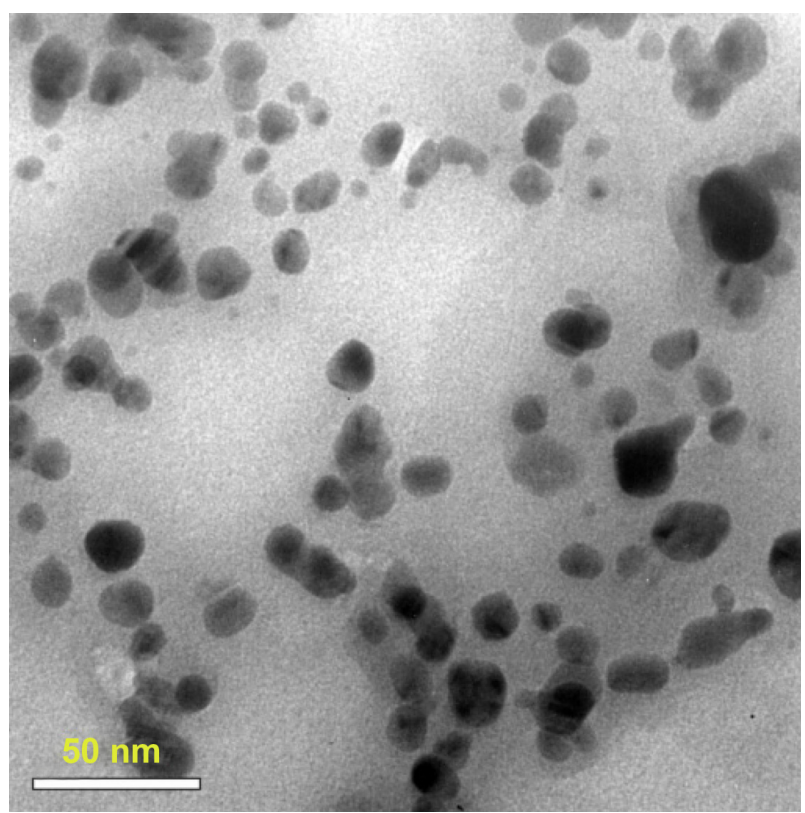

Figure SI TEM image of bare Ag nanoparticles synthesized by a chemical method.

Abbreviation: TEM, transmission electron microscopy.

A

Size distribution by intensity

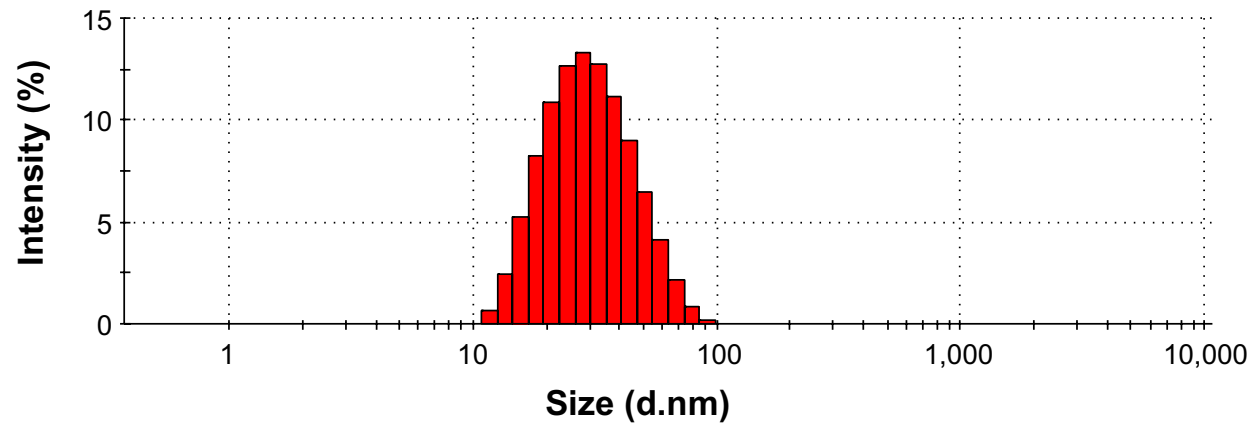

B

Zeta-potential distribution

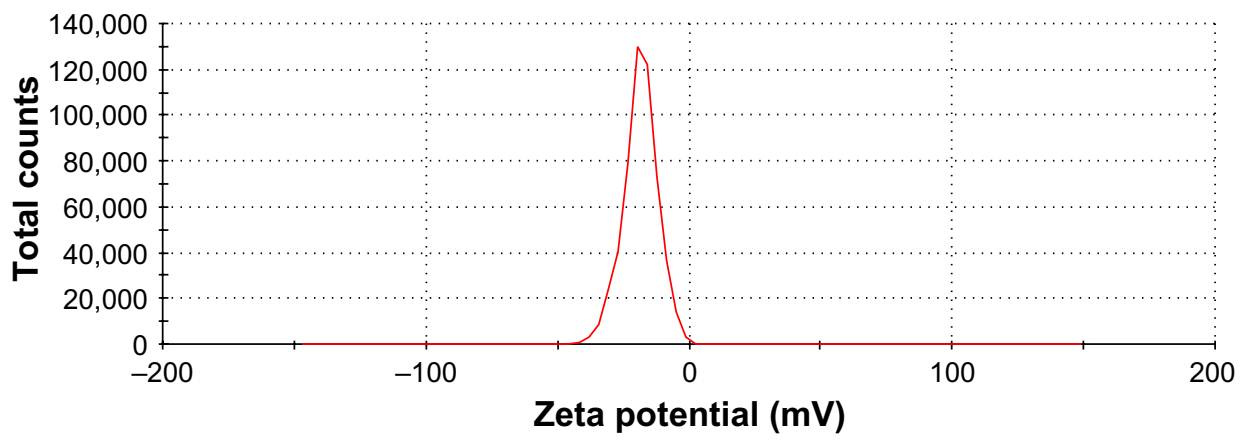

Figure S2 (A and B) DLS patterns. (A) Size distribution of bare AgNPs; (B) zeta-potential analysis of bare Ag nanoparticles. Abbreviation: DLS, dynamic light scattering. 


\section{Publish your work in this journal}

The International Journal of Nanomedicine is an international, peerreviewed journal focusing on the application of nanotechnology in diagnostics, therapeutics, and drug delivery systems throughout the biomedical field. This journal is indexed on PubMed Central, MedLine, CAS, SciSearch ${ }^{\circledR}$, Current Contents ${ }^{\circledR} /$ Clinical Medicine,

Journal Citation Reports/Science Edition, EMBase, Scopus and the Elsevier Bibliographic databases. The manuscript management system is completely online and includes a very quick and fair peer-review system, which is all easy to use. Visit http://www.dovepress.com/ testimonials.php to read real quotes from published authors.

Submit your manuscript here: http://www.dovepress.com/international-journal-of-nanomedicine-journal 